\title{
Deglutição e aspiração na Implantodontia
}

\author{
Deglutition and aspiration on Implantology \\ Deglución y aspiración en Implantología \\ Jéssica Lemos GULINELLI ${ }^{1}$ \\ Matheus de VITTO $^{1}$ \\ Ana Carolina de Almeida LIMA ${ }^{1}$ \\ Marcus KASAYA ${ }^{\mathbf{1}}$ \\ Pâmela Letícia SANTOS ${ }^{2}$
}

${ }^{1}$ Faculdade de Odontologia, USC Univ. Sagrado Coração, 17011-160 Bauru - SP, Brasil.

${ }^{2}$ Departamento de Ciências da Saúde, Faculdade de Odontologia, UNIARA Universidade de Araraquara, 14801-340 Araraquara - SP Brasil

\section{Resumo}

Introdução: O tratamento com implantes e próteses implanto-suportadas tem sido amplamente utilizado em áreas edêntulas, aumentando assim o risco de acidentes e complicações. Dentre os acidentes em implantodontia destaca-se A migração de implantes e componentes para a orofarínge ocasionando em deglutição ou aspiração. Objetivo: O presente trabalho teve como objetivo, abordar acidentes relacionados à deglutição e aspiração, focando no tratamento e prevenção dos mesmos, por meio de uma revisão da literatura e relato de dois casos clínicos, ilustrando as intercorrências. Resultados: Os acidentes de deglutição e aspiração, apesar não frequentes, podem acontecer em qualquer tratamento odontológico, sendo de extrema importância o emprego de métodos preventivos. Em geral são assintomáticos e o diagnóstico por imagem se faz necessário para localização do objeto e definição de plano de tratamento. Conclusão: A deglutição é um acidente de fácil tratamento, pois geralmente com dieta rica em fibras o objeto pode ser expelido. Já no caso de aspiração, o paciente deve ser encaminhado a um centro de emergência e submetido a uma laringoscopia ou broncoscopia.

Descritores: Deglutição; Sucção; Corpos Estranhos; Implantes Dentários.

\section{Abstract}

Background: Treatment with implant-supported implants and implants has been widely used in edentulous areas, thus increasing the risk of accidents and complications. Among the accidents in implant dentistry are the components and implants that penetrate the oropharynx, causing swallowing or aspiration. Aim: The present study had to objective, address accidents related to swallowing and aspiration, focusing on their treatment and prevention, through a review of the literature and a report of a clinical case. Results: The swallowing and aspiration accidents, although not frequent, can happen in any dental treatment, being of extreme importance preventive methods. In general, they are asymptomatic and the diagnosis by imaging is necessary to locate the object and define the treatment plan. Conclusion: Swallowing is an easy treat accident, since usually with a high fiber diet the object can be expelled. In the case of aspiration, the patient should be referred to an emergency center and submitted to a laryngoscopy or bronchoscopy.

Descriptors: Deglutition; Suction; Foreign Bodies; Dental Implants.

\section{Resumen}

Introducción: El tratamiento con implantes y prótesis implanto-soportadas ha sido ampliamente utilizado en áreas edéntulas, aumentando asî el riesgo de accidentes y complicaciones. Entre los accidentes en implantodoncia se destaca la migración de implantes y componentes para la orofarínge ocasionando en deglución o aspiración. Meta: El presente trabajo tuvo como objetivo, abordar accidentes relacionados con la deglución y aspiración, enfocándose en el tratamiento y prevención de los mismos, por medio de una revisión de la literatura y relato de dos casos clínicos, ilustrando las intercurrencias. Resultado: Los accidentes de deglución y aspiración, aunque no frecuentes, pueden ocurrir en cualquier tratamiento odontológico, siendo de extrema importancia métodos preventivos. En general son asintomáticos y el diagnóstico por imagen se hace necesario para localización del objeto y definición de plan de tratamiento. Conclusión: La deglución es un accidente de fácil tratamiento, pues generalmente con dieta rica en fibras el objeto puede ser expulsado. En el caso de aspiración, el paciente debe ser encaminado a un centro de emergencia y sometido a una laringoscopia o broncoscopia.

Descriptores: Deglución; Succión; Cuerpos Extraños; Implantes Dentales.

\section{INTRODUÇÃO}

Os implantes osseointegrados possuem alto índice de sucesso fato que acarreta na ampliação de suas indicações, na qualificação dos profissionais, biotecnologias envolvidas e diversidade do tratamento ${ }^{1}$. Entretanto, o número e a gravidade das complicações relacionadas aos implantes também aumentaram em proporções significativas ${ }^{2}$.

Dentre as complicações em implantodontia pode-se citar a deglutição e aspiração de componentes e implantes. De acordo com Silva et al. ${ }^{3}$ a passagem de corpos estranhos pela orofaringe durante $\mathrm{o}$ tratamento odontológico constitui um acidente que pode colocar em risco a vida do paciente. Nesses casos a conduta inicial consiste em avaliar se a via aérea não está obstruída, e encaminhar o paciente ao pronto atendimento ${ }^{4}$. No hospital, o procedimento realizado pela equipe médica abrange a localização do instrumental por meio de exames radiográficos torácicos e/ ou abdominais, exceto para os componentes radiopacos, que utilizam-se as broncoscopia ou tomografia computadorizada ${ }^{4}$.

Os corpos estranhos mais comuns de penetrarem pela orofaringe durante qualquer tratamento dentário incluem instrumentais endodônticos, brocas, pinos, dentes, braquetes ortodônticos, restaurações ou componentes dos implantes ${ }^{5}$. Os instrumentos utilizados para tratamento com implantes bucais são geralmente pequenos, e muitos componentes do implante são ainda menores. Como a saliva é escorregadia, ocasionalmente, esses instrumentos podem escorregar das mãos do operador. Destes acidentes, $87 \%$ dos instrumentos são ingeridos, e $13 \%$ aspirados ${ }^{6}$.

Os instrumentos odontológicos ingeridos são 80 a $90 \%$ eliminados pelo corpo de forma natural ao longo de alguns dias ou semanas. Todavia, em alguns casos podem resultar em sérias complicações, incluindo hemorragia, infecção, obstrução intestinal e até mesmo perfuração ${ }^{4}$. Os sintomas dessas 
complicações são relatados como dor abdominal ou vômitos. Assim se faz necessário o acompanhamento radiográfico desse paciente para verificar a localização do instrumento, e se alguma complicação como perfuração de algum órgão em seu trajeto viabilizando uma intervenção cirúrgica nesses casos ${ }^{4}$.

Já nas situações de aspiração de um corpo estranho as medidas geralmente são imediatas, pois pode gerar dispneia, asfixia, edema de laringe, perfuração pulmonar e até ocasionar o paciente a óbito. O risco de ocorrência desse acidente é maior em crianças, idosos, obesos, alcoólatras, deficientes mentais e usuários de drogas de ação central. Para esses casos o tratamento de escolha é a broncoscopia para remoção do objeto aspirado ${ }^{7,8}$.

Entretanto, esses acidentes podem ser evitados na maioria das vezes, principalmente quando o profissional adota condutas preventivas, como amarrar determinados objetos com fio dental ${ }^{9}$, utilizar dique de borracha sempre que possível, ${ }^{4}$ tela de gaze na orofaringe, manejo adequado do paciente $\mathrm{e}$ verificar antes e depois todos os instrumentos utilizados ${ }^{7}$.

Diante de tais condições, além da conduta supracitada, o profissional deve ter cuidados judiciais incluindo manter o paciente informado sobre o tipo de manobra que será efetuada, sobretudo aquelas que têm o risco aumentado de acidente e documentar todo o procedimento (data, objeto envolvido, técnicas de intervenção, cuidados médicos e cópias das radiografias). Assim, o objetivo do presente estudo foi abordar os acidentes em implantodontia relacionadas à deglutição e aspiração, por meio de caso clínico.

\section{CASO CLÍNICO}

\section{Caso 1: Aspiração}

Paciente do gênero masculino, com 70 anos de idade, compareceu para tratamento de implantes nas regiões dos elementos 36 e 46. Na história médica o paciente relatou não apresentar doenças sistêmicas. No dia 08/03/2013 foram realizadas as cirurgias e ocorreu o procedimento normal sem acidentes e complicações. No 23/07/2013 foi marcada a instalação das próteses nessas regiões, e durante a instalação da prótese o paciente engoliu acidentalmente a chave de implante quadrada do kit protético Neodent. Clinicamente o paciente não apresentou nenhuma sintomatologia e então foram solicitados radiografia de tórax e o diagnóstico foi aspiração da chave (Figura 1). Para realizar o tratamento do paciente, o mesmo foi encaminhado ao Hospital e a chave foi removida com broncofibroscópio com sedação endovenosa; sendo liberado no mesmo dia. No dia 25/07/2013 o paciente voltou para controle, apresentando-se em ótimo estado de saúde e sem nenhuma queixa.

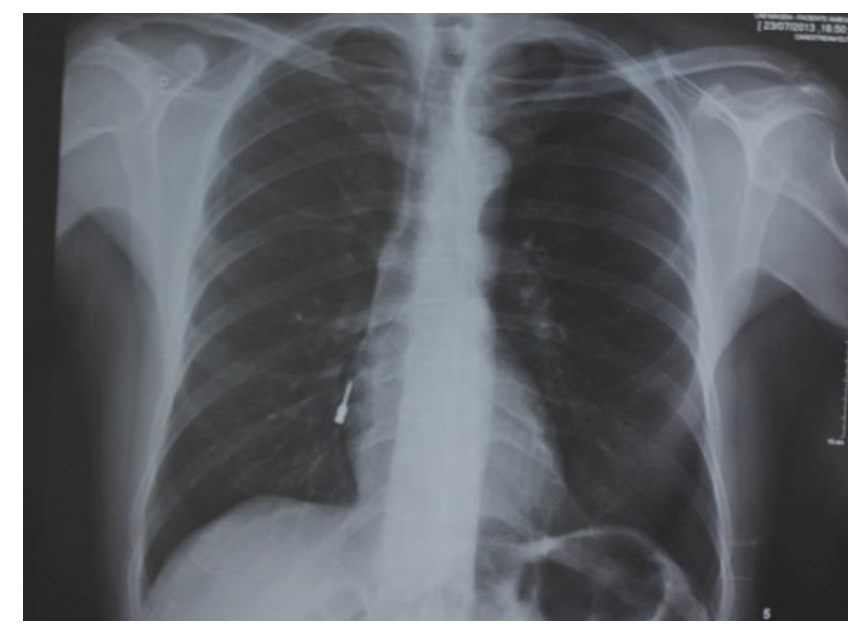

Figura 1: Aspiração de chave digital de implante alojada na região de tórax.

\section{Caso 2: Deglutição}

Paciente do gênero feminino, com 55 anos de idade, compareceu para tratamento de implantes nas regiões dos elementos 14 a 16. Na história médica o paciente relatou não apresentar doenças sistêmicas. No dia 22/10/2014 foi realizado o procedimento cirúrgico e paciente deglutiu o dispositivo medidor de altura de intermediários protéticos de implante cone morse. Foram solicitados radiografias de tórax e o diagnóstico foi deglutição da chave (Figuras 2 e 3). Para realizar o tratamento do paciente, o mesmo foi orientado quanto à alimentação (alimentos ricos em fibras) e bastante água. No dia 24/10/2014 o paciente voltou para controle, relatando ter expelido o instrumental e apresentando-se em ótimo estado de saúde e sem nenhuma queixa.

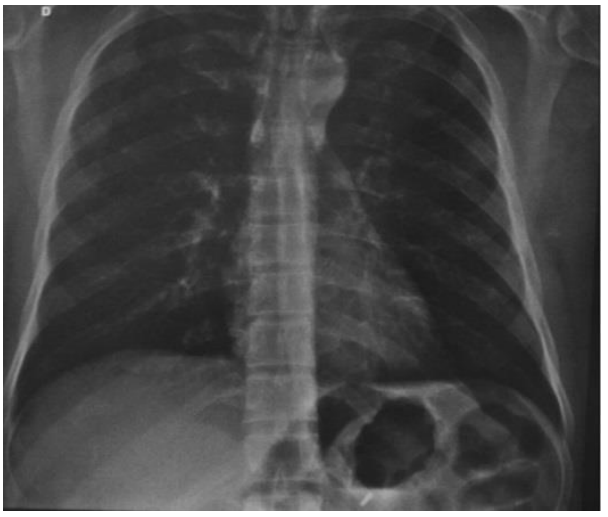

Figura 2: Deglutição de dispositivo medidor de altura de intermediários protéticos de implante cone morse.

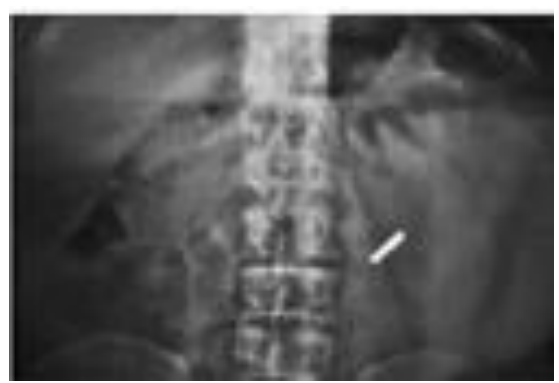

Figura 3: Deglutição de dispositivo medidor de altura de intermediários protéticos de implante cone morse. 


\section{DISCUSSÃO}

Os corpos estranhos que são acidentalmente deglutidos ou aspirados e não tratados corretamente, podem causar complicações graves, pois devido à agudeza do instrumento existe o risco de perfurações das estruturas anatômicas ${ }^{6}$. Esses acidentes podem ocorrer devido à imprudência do profissional, mau uso dos instrumentos e visualização inadequada do campo operatório, além disso, esterilizações sucessivas dos instrumentais como brocas e chaves de implante, diminuem sua vida útil levando a um maior risco de fraturas e, consequentemente, a acidentes de deglutição ou aspiração ${ }^{10}$.

O diagnóstico da aspiração e deglutição de corpos estranhos depende dos sinais e sintomas exibidos pelo paciente, bem como da demonstração radiográfica da presença do corpo estranho gastrointestinal ou no trato respiratório. A deglutição é quase sempre assintomática e sem sequelas, porém, um corpo estranho que entra na faringe e então passa pelo esôfago pode produzir tosse, ânsia, dor no pescoço, salivação excessiva, sensação de algo entalado na garganta e dificuldade em engolir; já os corpos estranhos que entram no trato respiratório irão se deslocar conforme o tamanho, objetos grandes irão se alojar perto ou nas cordas vocais, enquanto que objetos pequenos mais frequentemente passam para o brônquio direito $^{11}$. E assim, a aspiração acidental de instrumentos dentários pode ser um evento ainda mais grave do que a deglutição e deve ser sempre tratado como uma situação de emergência ${ }^{7}$.

O diagnóstico por imagem é de suma importância para a confirmação do local onde o instrumento está alojado. Para verificar se o objeto foi ingerido ou aspirado, um exame radiográfico de abdômen pode ser útil, caso não seja visualizado no abdômen, pode-se lançar mão de radiografias de tórax PA e lateral ${ }^{12}$. Haliloglu et al. ${ }^{13}$ sugeriram que quando a radiografia de tórax não possuir alterações da normalidade e o diagnóstico clínico sugerir corpo estranho aspirado, tomografia helicoidal e broncoscopia virtual podem ser consideradas.

Já autores como Sá-Lima et al. ${ }^{14}$ recomendaram tomografia computadorizada para casos de inalação ou deglutição de PPRs, especialmente em indivíduos mais velhos $\mathrm{e}$ deficientes mentais. Por outro lado, Huang et al. ${ }^{15}$ concluíram que a tomografia computadorizada tridimensional foi mais sensível que as radiografias de tórax em detectar a presença de corpos estranhos aspirados em crianças. O exame radiográfico é obrigatório para o diagnóstico diferencial da localização, natureza e tamanho do corpo estranho, podendo iniciar pela tomada de tomografia computadorizada ou sequência de radiografias que incluam anteroposterior e lateral do tórax, pescoço lateral e radiografias de abdômen realizadas para complementar avaliação da nasofaringe ao reto ${ }^{6}$.
Em casos acidentais de deglutição e aspiração, consulta médica e radiografias são essenciais para confirmação e para o planejamento da intervenção ${ }^{11}$. Nos casos de deglutição a avaliação radiográfica e uma dieta rica em fibras é o protocolo inicial, pois pode ajudar que o corpo estranho seja eliminado nas fezes ${ }^{6,16}$. Ramalho-Ferreira et al. ${ }^{17}$ complementam que no caso de deglutição de objeto sem ponta perfuro-cortante poderá ocorrer trânsito por todo o trato gastrintestinal, com posterior eliminação.

Por outro lado, se o paciente relatar dor abdominal e/ou presença de sangue nas fezes, pode ser sinal de perfuração intestinal, retenção do instrumento ou de obstrução, e nesse caso, a remoção cirúrgica é indicada, por meio de acesso abdominal para facilitar a visualização e a remoção do instrumento através de broncoscopia ou endoscopia digestiva ${ }^{6}$. Para Pull ter Gunne e Wismeijer ${ }^{5}$ esperase que o objeto estranho irá sair do corpo de forma natural, uma vez que a maioria $(80 \%$ a $90 \%)$ de objetos estranhos que atingem o trato gastrointestinal vai passar espontaneamente, de $10 \%$ a $20 \%$ irá requerer intervenção não cirúrgica, e $1 \%$ ou inferior necessitarão de cirurgia.

No caso clínico relatado por Pingarrón Martín et al. ${ }^{7}$ de paciente com suspeita de ingestão de uma chave com conexão fenda de implantes, as radiografias de tórax nas projeções póstero-lateral mostraram um instrumento dental radiopaco impactado no brônquio principal direito inferior, realizaram uma broncoscopia rígida sob anestesia geral que foi bem sucedida para remover o instrumento odontológico. Também no caso clínico relatado por Souza et al. ${ }^{6}$ em que durante a cirurgia de implante, a chave com conexão fenda foi involuntariamente ingerida pelo paciente, as radiografias de peito frontal e abdominal confirmaram que o instrumento estava localizado no trato gastrointestinal; o paciente foi submetido a uma dieta rica em fibras, numa tentativa de eliminar o instrumento fisiologicamente sem o uso de laxantes, como isso não aconteceu optou-se por realizar uma intervenção cirúrgica para remover o instrumento, pois a chave com conexão fenda estava obstruindo a válvula ileocecal, o procedimento cirúrgico foi feito sob anestesia geral, sendo realizada como uma laparotomia exploradora, seguido por uma colostomia de $1 \mathrm{~mm}$, imediatamente após os tecidos foram libertados e os instrumentais impactados removidos do cólon transverso. Por outro lado, Kim e $\mathrm{Ahn}^{18}$, em paciente com ingestão de uma chave com conexão fenda e pilar de cicatrização durante cirurgia de implantes, realizaram broncoscopia sob sedação consciente e utilizaram micropinças para remover os instrumentos. Os autores concluíram que a broncoscopia é o procedimento mais recomendado para a remoção do corpo estranho e isto pode ser 
realizado sob sedação consciente.

Autores como Ramalho-Ferreira et al. ${ }^{17}$ sugeriram que se o objeto for pequeno e for aspirado, obstruindo parcialmente as vias aéreas o paciente deverá ser imediatamente conduzido a um centro de emergência, recebendo oxigênio suplementar durante a transferência, onde deverá ser submetido a uma laringoscopia ou broncoscopia. Se houver uma obstrução maior das vias aéreas impedindo a respiração, dois procedimentos poderão ser realizados: compressão do abdômen ou a manobra de Heimlich. No caso clínico relatado por Deliberador et al. ${ }^{19}$ durante moldagem para a construção da prótese implantossuportada, o paciente acidentalmente aspirou a chave com conexão fenda do implante. $\mathrm{O}$ objeto foi encontrado no lóbulo do brônquio direito inferior, exigindo para sua remoção ambiente hospitalar por meio de broncoscopia, sob anestesia geral.

Contudo, o emprego de medidas preventivas ainda é a melhor opção para evitar complicações de deglutição e aspiração de instrumentos odontológicos $^{7}$. As intercorrências como deglutição ou aspiração devem ser evitadas por meio da colocação de uma compressa na orofaringe, mantida por uma pinça durante a manipulação de pequenos instrumentos que, podem ainda ser amarrados ao fio de sutura estéril, ou mesmo ao fio dental em procedimentos que dispensem a autoclavagem ${ }^{16,19}$. Para Coser et al. ${ }^{10}$ o dique de borracha é o meio mais simples e efetivo na prevenção contra deglutição e aspiração de corpos estranhos, por proporcionar um campo operatório limpo e seco, melhorando a visibilidade e o acesso, além disso, protege o paciente recobrindo os lábios, bochechas e tecido gengival, e ainda previne o movimento da língua no campo operatório. No caso de chaves de fenda e pequenos instrumentos usados em implantes, os mesmos podem ser amarrados por um pedaço de fio dental.

Outro fator importante é o posicionamento da cabeça do paciente durante determinados procedimentos, e diante da possibilidade real de que determinados objetos fiquem alojados na orofaringe, sempre que houver condições a cabeça do paciente deverá estar voltada para a lateral, permitindo assim que o objeto se acomode nas laterais da cavidade bucal e não na orofaringe ${ }^{9}$. Além disso, antes de usar instrumentos e brocas, devem-se analisar os sinais de uso e corrosão que são acelerados pelos processos de assepsia, devendo ser feito um controle do tempo de uso e limitar a reutilização de brocas ${ }^{11}$. Como não há consenso no tratamento de acompanhamento dos pacientes após esses eventos, há uma necessidade de diretrizes claras para os dentistas ${ }^{5}$.

\section{CONCLUSÃO}

Com base na literatura consultada pode-se concluir que acidentes de deglutição e aspiração, apesar não muito frequentes, podem acontecer em qualquer tratamento odontológico, e quando isso acontece o diagnóstico por imagem, por meio de radiografias e tomografias se fazem necessários para localizar o objeto. A deglutição é um acidente mais fácil de ser tratado, pois geralmente, com dieta rica em fibras, o objeto pode ser expelido. Nos casos de aspiração, por outro lado, o paciente deve ser encaminhado a um centro de emergência e submetido a uma laringoscopia ou broncoscopia.

\section{REFERENCIAS}

1. Pinto AVS. Fatores de risco na terapêutica com implantes osseointegrados [dissertação]. Campinas: Universidade Castelo Branco; 2000.

2. Lourenço SV, Morano-Júnior M, Daruge-Júnior E. Complicações cirúrgicas e protéticas em implantodontia. Rev Odonto Ciênc. 2007;22(58):352-58.

3. Silva RF, Ferreira GM, Leles JLR, Iwaki-Filho L, Durege-Junior E. Passagem de corpos estranhos pela orofaringe durante tratamento odontológico: abordagem clínica e odontolegal. Rev Cir Traumatol Buco-Maxilo-Fac. 2009;9(3):55-62.

4. Oncel M, Apiliogullari B, Cobankara FK, Apiliogullari S. Accidental swallowing of the head of a dental mirror: report of a rare case. $\mathbf{J}$ Dent Sci. 2012;7(2):199-202.

5. Pull ter Gunne L, Wismeijer D. Accidental ingestion of an untethered instrument during implant surgery. Int J Prosthodont. 2014;27(3):277-78.

6. Souza JG, Schuldt-Filho G, Pereira-Neto AR, Lyra HF Jr, Bianchini MA, Cardoso AC. Accident in implant dentistry: involuntary screwdriver ingestion during surgical procedure. A clinical report. J Prosthodont. 2012;21(3):191-93.

7. Pingarrón Martín L, Morán Soto MJ, Sánchez Burgos R, Burgueño García M. Bronchial impaction of an implant screwdriver alter accidental aspiration: report a case and revision of the literature. Oral Maxillofac Surg. 2010;14(1):43-7.

8. Eroglu O, Algan-Kaya H, Coskun F. A potentially fatal complication that may occur during dental treatment: "foreign body aspiration". Pan Afr Med J. 2015;20:36.

9. Silva RF, Prado FB, Portilho CDM, Silva RF, Daruge-Júnior E. Orientações clínicas e éticas em caso de deglutição de corpo estranho durante atendimento odontológico. RSBO. 2010;7(3):3549.

10.Coser S, Estrá $T$, Muraoka M, Martins LP, Brugnara E, Pinto JR, et al. Projeção e aspiração de corpos estranhos para as vias respiratórias e digestivas. UNOPAR Cient Ciênc Biol Saúde. 2000;2(1):113-21.

11.Hodges ED, Durhan TM, Stanley RT. 
Management of aspiration and swallowing incidents: a review of the literature and report of cases. ASDC J Dent Child. 1992;59(6):413-19.

12. Fields RT, Schow SR. Aspiration and ingestion of foreign bodies on oral and maxillofacial surgery: a review of the literature and report of five cases. $\mathbf{J}$ Oral Maxillofac Surg.1998;56(9):1091-98.

13. Haliloglu M, Ciftci AO, Oto A, Gumus B, Tanyel FC, Senocak ME et al. CT virtual bronchoscopy in the evaluation of children with suspected foreign body aspiration. Eur $\mathrm{J}$ Radiol. 2003;48(2):188-92.

14. Sá-Lima JR, Raldi FV, Murilo-Santos L, FerrerCarneiro RG, Michida SM de A. Deglutição acidental de prótese parcial removível: relato de caso. Rev Int Cir Traumatol Bucomaxilofacial.2005;3(11/12):143-46.

15.Huang HJ, Fang HY, Chen HC, Wu CY, Cheng CY, Chang CL. Three-dimensional computed tomography for detection of tracheobronchial foreign body aspiration in children. Pediatr Surg Int. 2008;24(2):157-60.

16. Costa JL. Complicações e fracassos na implantodontia [monografia]. Manaus: Instituto Brasiliense de Pós-Graduação; 2010.

17.Ramalho-Ferreira G, Faverani LP, Gomes PCM, Assunção WG, Garcia-Júnior IR. Complicações na reabilitação bucal com implantes osseointegráveis. Rev Odontol Araçatuba. 2010;31:51-5.

18. Kim A, Ahn KM. Endoscopic removal of an aspirated healing abutment and screwdriver under conscious sedation. Implant Dent. 2014;23(3):250-52.

19.Deliberador TM, Marengo G, Scaratti R, Giovanini AF, Zielak JC, Baratto-Filho F. Accidental aspiration in a patient with Parkinson's disease during implant-supported prosthesis construction: a case report. Spec Care Dentist. 2011;31(5):156-61.

\section{CONFLITO DE INTERESSES}

Os autores declaram não haver conflitos de interesse.

AUTOR PARA CORRESPONDENCIA

Pâmela Letícia dos Santos

pamelalsantos@hotmail.com

Submetido em 10/05/2018

Aceito em 27/06/2018 